\title{
Does NIDDM increase the risk for coronary heart disease similarly in both low- and high-risk populations?
}

\author{
M.Laakso ${ }^{1}$, T. Rönnemaa ${ }^{2,3}$, S. Lehto ${ }^{1}$, P.Punkka ${ }^{3}$, V.Kallio ${ }^{3}$, K.Pyörälä ${ }^{1}$ \\ ${ }^{1}$ Department of Medicine, Kuopio University Hospital, Kuopio, Finland \\ ${ }^{2}$ Department of Medicine, Turku University Central Hospital, Turku, Finland \\ ${ }^{3}$ Rehabilitation Research Centre of the Social Insurance Institution, Turku, Finland
}

\begin{abstract}
Summary Finland has marked regional differences in the occurrence of coronary heart disease (CHD). Although the causes for these differences in CHD mortality and morbidity in the Finnish population are unknown, it offers an excellent opportunity to investigate the effects of non-insulin-dependent diabetes mellitus (NIDDM) on CHD risk in two populations differing significantly with respect to the occurrence of CHD. Therefore, we carried out a 7-year prospective population-based study including a large number of patients with NIDDM (East Finland: 253 men and 257 women; West Finland: 328 men, 221 women) and corresponding non-diabetic subjects (East Finland: 313 men, 336 women; West Finland: 325 men, 399 women). In both study populations the presence of NIDDM increased significantly the risk for CHD events (CHD mortality or all CHD events including
\end{abstract}

CHD mortality or non-fatal myocardial infarction). Diabetic men had 3-4 fold higher and diabetic women 8-11-fold higher risk for CHD than corresponding non-diabetic subjects. Both non-diabetic and diabetic subjects had odds ratios (East vs West) for CHD events of about 2 indicating a similar EastWest difference in the CHD risk. Regional difference was quite similar in men and women. These results imply that factors related to NIDDM, independently of conventional risk factors and the occurrence of atherothrombosis in the background population, must play a major role in the pathogenesis of atherosclerotic vascular disease in NIDDM diabetes.

Key words Coronary heart disease, non-insulin-dependent diabetes mellitus. [Diabetologia (1995) 38: 487-493].
Several previous population-based studies have indicated that subjects with non-insulin-dependent diabetes mellitus (NIDDM) are at increased risk of developing atherosclerotic vascular disease in all its manifestations [1]. The most serious of these, coronary heart disease (CHD), is the leading cause of death among the patients with NIDDM. The reasons for this excess risk have remained largely unknown and are not completely explained by adverse changes in cardiovascular risk factors associated with the

Received: 6 April 1994 and in revised form: 21 October 1994

Corresponding author: Dr. M.Laakso, Department of Medicine, Kuopio University Hospital, SF-70210 Kuopio, Finland Abbreviations: CHD, Coronary heart disease; NIDDM, non-insulin dependent diabetes mellitus; MI, myocardial infarction. diabetic state. Particularly striking in these studies has been the finding that NIDDM abolishes the protective effect of female gender with respect to the risk for CHD [1]. Consequently, the relative impact of diabetes in women substantially exceeds the corresponding risk in men.

There are marked differences between ethnically homogeneous populations in the occurrence of $\mathrm{CHD}$ which are mainly explained by life-style-related risk factors, in particular by diet-related between-population differences in total cholesterol levels [2, 3]. Furthermore, trends in CHD mortality and morbidity within populations parallel changes in life-style-related risk factors giving strong support to the concept of powerful environmental determinants of the occurrence of CHD within populations [4].

How do marked differences in the occurrence of CHD between populations become reflected in the 
occurrence of CHD in diabetic individuals in these populations? The answer to this question is not known. However, this issue is of particular interest because it gives important information on the role of diabetes in the pathogenesis of CHD. For example, if diabetic subjects have a similar or higher relative excess of CHD in a population with a high prevalence of CHD in non-diabetic subjects than in a population with a lower prevalence of CHD in non-diabetic subjects, this implies that the diabetic state itself or factors related to it must be highly important for the risk of CHD because the diabetic state increases the risk irrespective of conventional risk factor levels.

Finland has a marked regional difference in the occurrence of CHD. The mortality and morbidity of CHD in East Finland has been twofold higher compared to West Finland $[2,5,6]$. The reasons for this regional difference are not completely understood but they are partly due to elevated cardiovascular risk factor levels in subjects living in East Finland [7]. The Finnish population is genetically quite homogeneous [8] although it is not completely excluded that part of the East-West difference in CHD could be due to currently unknown genetic factors. Whatever the causes of the marked regional differences in CHD mortality and morbidity in the Finnish population might be, they offer an excellent opportunity to investigate the effects of NIDDM on CHD risk in two populations differing significantly with respect to the occurrence of CHD. Therefore, our 7-year prospective population-based study including a large number of patients with NIDDM was designed to investigate the following questions: 1) is the effect of NIDDM on CHD risk similar in two populations with high (East Finland) and relatively low (West Finland) occurrence of CHD? 2) is the effect of NIDDM on CHD risk similar in both sexes in these two populations?

\section{Subjects and methods}

Patients with NIDDM. All diabetic patients in Finland who need drug therapy receive it free of charge according to the Sickness Insurance Act. The Social Insurance Institution maintains a central register of diabetic subjects receiving drug reimbursement (coverage $>98 \%$ ). Based on this register, we identified all diabetic patients, aged 45-64 years, who were born and living in the Kuopio University Hospital district (East Finland) and in the Turku University Central Hospital district (West Finland). The formation of the final patient population consisting of 510 diabetic subjects (253 men, 257 women) who participated in this study in East Finland (participation rate $83 \%$ ) and 549 diabetic subjects ( 328 men, 221 women) who participated in the study in West Finland (participation rate $79 \%$ ) has been previously described in detail [9]. Insulin-dependent diabetes was excluded in all insulin-treated patients by $\mathrm{C}$-peptide measurements. All diabetic patients included in the final study population showed a plasma C-peptide concentration of at least $0.20 \mathrm{mmol} / 1$ at $6 \mathrm{~min}$ after injection of $1 \mathrm{mg}$ of intravenous glucagon. A cutoff point of $0.20 \mathrm{nmol} / 1$ was chosen because post-glucagon C-peptide values below this limit have been shown to be associated with the occurrence of ketoacidosis in insulin-treated diabetic subjects [10]. None of the patients classified as having NIDDM according to the World Health Organisation (WHO) criteria [11], and included in the final study population, had a history of ketoacidosis. Of the 510 NIDDM patients from East Finland, 46 men and 38 women were treated with diet only, 183 men and 175 women with oral hypoglycaemic drugs, and 24 men and 44 women with insulin. Of 549 NIDDM patients from West Finland, 46 men and 17 women were treated with diet only, 227 men and 177 women with oral hypoglycaemic drugs, and 55 men and 27 women with insulin. The age of diabetic men from East Finland was $56.8 \pm 0.3$ and from West Finland $57.2 \pm 0.3$ years, and the age of diabetic women from East Finland was $58.9 \pm 0.3$ and from West Finland $58.7 \pm 0.3$ years.

Non-diabetic subjects. A random control-population sample of subjects whose place of birth was in the Kuopio University Central Hospital district (East Finland) or in the Turku University Central Hospital district (West Finland) and who were living in these areas was taken from the population register containing all subjects aged 45-64 years. The formation of these non-diabetic samples has been previously published [9]. Participation rates were $79 \%$ in East Finland and $85 \%$ in West Finland. The final study population consisted of 649 subjects (313 men, 336 women) in East Finland and 724 subjects ( 325 men, 399 women) in West Finland. The age of non-diabetic men from East Finland was 53.8 \pm 0.3 and from West Finland $54.5 \pm 0.3$ years, and the age of non-diabetic women from East Finland $54.6 \pm 0.3$ and from West Finland $54.0 \pm 0.3$ years.

This study was approved by the ethics committee of the Kuopio University Central Hospital

Study programme and methods at baseline examination from 1982-1984. The study programme for both diabetic and nondiabetic subjects was carried out during one outpatient visit to the Clinical Research Unit of the University of Kuopio or the Rehabilitation Research Centre of the Social Insurance Institution in Turku. These methods have been previously described in detail [9]. The visit included an interview on the history of chest pain symptoms suggestive of CHD, smoking, alcohol, intake, physical activity and the use of drugs. All medical records of those subjects who reported at the interview that they had been admitted to the hospital on the basis of chest pain symptoms were reviewed. Review of the medical records was performed by two of us (M. L. in Kuopio and T.R. in Tur$\mathrm{ku}$ ) after a careful standardization of the methods between the reviewers. The WHO criteria for verified definite and possible myocardial infarction (MI) based on chest pain symptoms, ECG changes, and enzyme determinations were used in the ascertainment of the diagnosis of previous MI [12]. A patient was classified as hypertensive if he/she was receiving drug treatment for hypertension or if his/her systolic blood pressure was at least $160 \mathrm{~mm} \mathrm{Hg}$ or diastolic blood pressure $95 \mathrm{~mm} \mathrm{Hg}$.

Biochemical methods. All laboratory specimens were drawn after a 12 -h fast at 08.00 hours. Fasting plasma glucose was determined by the glucose oxidase method (Boehringer Mannheim, Mannheim, Germany). The plasma C-peptide response to glucagon was determined according to the method of Faber and Binder [13]. C-peptide was measured by radioimmunoassay (antibody M 1230, Novo, Bagsvaerd, Denmark). Serum lipids and lipoproteins were determined from fresh serum samples drawn after a 12-h overnight fast. Serum total cholesterol and triglycerides were assayed by automated enzymatic meth- 
Table 1. Age-adjusted characteristics of study populations by sex and area of residence

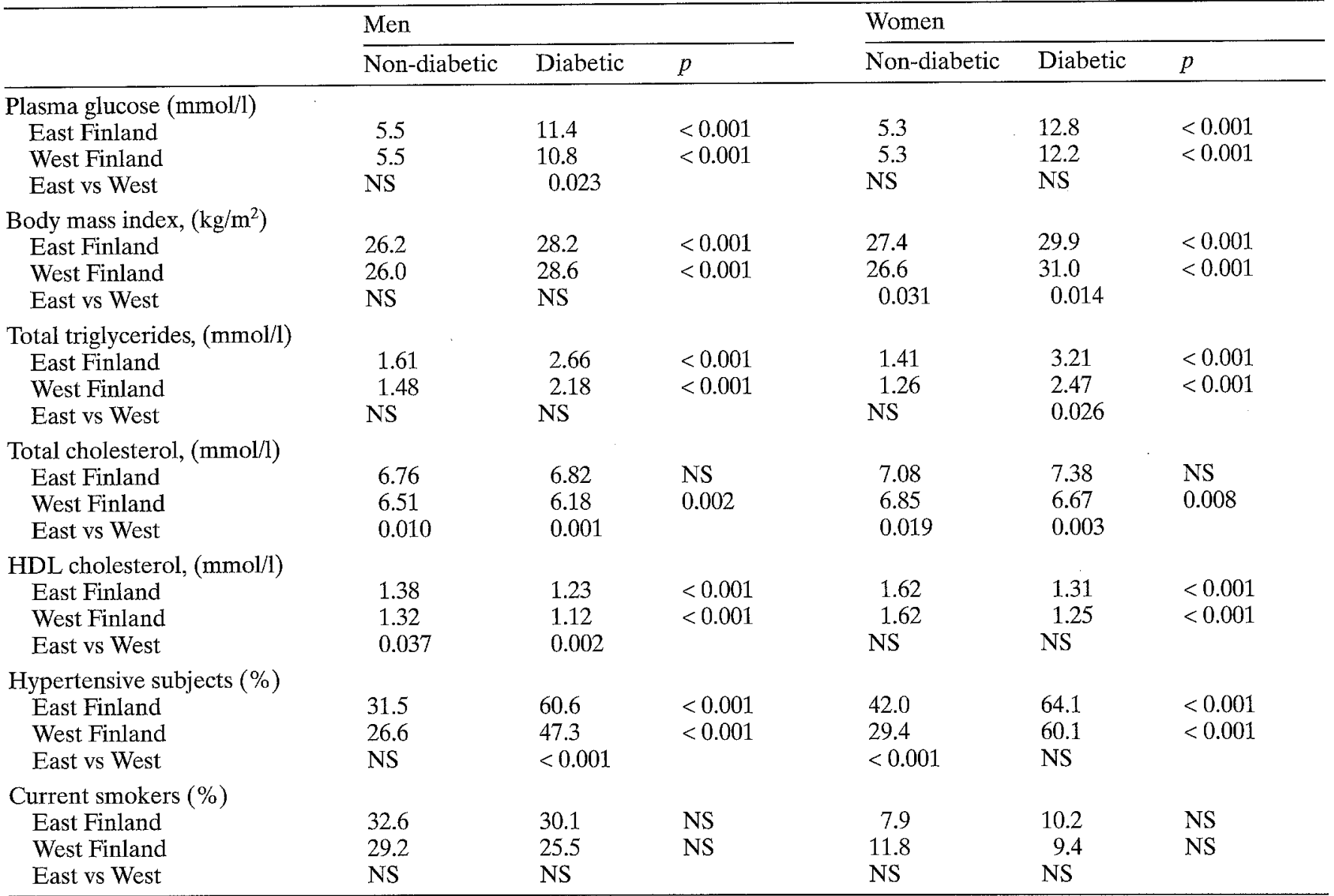

ods (Boehringer). Serum HDL cholesterol was determined enzymatically after precipitation of low-density and very-lowdensity lipoprotein with dextran sulfate- $\mathrm{MgCl}_{2}$ [14].

Collection of the follow-up data. In 1990 a postal questionnaire containing questions about hospitalization because of acute chest pain and symptoms suggestive of stroke was sent to every surviving participant of the original study cohort. All medical records of those subjects who died between baseline examination and 31 December 1989 or who reported in the questionnaire that they had been admitted to the hospital on the basis of chest pain symptoms between the baseline examination and 31 December 1989, were reviewed by one of us (S.L.). All deaths were ascertained from the Social Insurance Institution's register which has a coverage of $100 \%$ of the vital status of all diabetic subjects receiving drug reimbursement. Furthermore, the completeness of the information for CHD events was ascertained by a review of computerized hospital discharge data files. Using these data files a list of hospital discharge diagnoses was produced for the subjects who participated in the baseline study. Hospital records of subjects found in this list and having codes $410-414$ of the International Classification of Diseases 9 were checked for CHD events. Therefore, it is not likely that we have missed a significant number of nonfatal CHD events. The WHO criteria for verified and possible MI based on chest pain symptoms, ECG changes, and enzyme determinations were used in the ascertainment of the diagnosis of MI similarly to the baseline study [12]. Copies of death certificates of those patients who had died were obtained from the files of the Central Statistical Office of Finland. In the final classification of the causes of death hospital records and autopsy records were also used, if available. The mortality data included in the present article are total mortality, mortality from cardiovascular diseases (International Classification of Diseases 9, codes 390-458) and mortality from CHD (codes 410-414).

\section{Statistical analysis}

Statistical analyses of the data were carried out at the Rehabilitation Research Centre of the Social Insurance Institution, Turku, with OSIRIS IV (1981) and SAS computer programs and programs developed at the Social Insurance Institution.

The results for continuous variables are expressed as means \pm SEM. The differences between the groups were assessed by the $\mathrm{X}^{2}$ test, or Student's two-tailed $t$-test for independent samples, when appropriate. Age-adjusted prevalence rates were calculated by direct standardization methods [15] over four 5-year age groups with the total 1980 Finnish population aged 45-64 years as a standard population. Linear model was applied in the age adjustment of continuous variables [16]. The $F$-test was used in statistical testing of differences or prevalences or mean values of continuous variables controlling for the effect of age with 5-year age groups [16]. Odds ratios and their $95 \%$ confidence limits were calculated according to Gart [17]. Odds ratios were used in this follow-up study as an estimate of the relative risk of a study population for CHD compared with a control population. In statistical analyses CHD events were classified as follows: 1) CHD death, 
Table 2. Age-adjusted risks of total, cardiovascular and coronary heart disease (CHD) mortality and all CHD events by sex and area of residence

\begin{tabular}{|c|c|c|c|c|c|c|}
\hline & \multicolumn{3}{|l|}{ Men } & \multicolumn{3}{|l|}{ Women } \\
\hline & Non-diabetic & Diabetic & $p$ & Non-diabetic & Diabetic & $p$ \\
\hline \multicolumn{7}{|c|}{ All subjects } \\
\hline East Finland & 11.6 & 26.6 & $<0.001$ & 4.5 & 20.3 & $<0.001$ \\
\hline West Finland & 6.2 & 23.2 & $<0.001$ & 2.2 & 16.5 & $<0.001$ \\
\hline East vs West & 0.012 & NS & & NS & NS & \\
\hline \multicolumn{7}{|c|}{ Cardiovascular mortality (\%) } \\
\hline East Finland & 6.8 & 20.2 & $<0.001$ & 0.6 & 14.0 & $<0.001$ \\
\hline West Finland & 4.1 & 15.5 & $<0.001$ & 0.3 & 8.6 & $<0.001$ \\
\hline East vs West & NS & 0.014 & & NS & 0.037 & \\
\hline \multicolumn{7}{|c|}{ CHD mortality (\%) } \\
\hline East Finland & 4.5 & 17.8 & $<0.001$ & 0.6 & 8.2 & $<0.001$ \\
\hline West Finland & 3.2 & 12.7 & $<0.001$ & 0 & 5.6 & $<0.001$ \\
\hline East vs West & NS & 0.012 & & NS & NS & \\
\hline \multicolumn{7}{|c|}{ All CHD events (\%) } \\
\hline East Finland & 10.0 & 29.9 & $<0.001$ & 1.8 & 17.6 & $<0.001$ \\
\hline West Finland & 5.7 & 20.1 & $<0.001$ & 0.5 & 11.5 & $<0.001$ \\
\hline East vs West & 0.033 & 0.001 & & NS & NS & \\
\hline \multicolumn{7}{|c|}{ MI free subjects } \\
\hline \multicolumn{7}{|c|}{ CHD mortality (\%) } \\
\hline East Finland & 3.3 & 13.1 & $<0.001$ & 0.6 & 7.8 & $<0.001$ \\
\hline West Finland & 2.0 & 8.8 & $<0.001$ & 0 & 5.1 & $<0.001$ \\
\hline East vs West & NS & NS & & NS & NS & \\
\hline \multicolumn{7}{|c|}{ All CHD events (\%) } \\
\hline East Finland & 9.4 & 23.9 & $<0.001$ & 1.5 & 16.5 & $<0.001$ \\
\hline West Finland & 4.1 & 16.0 & $<0.001$ & 0.5 & 10.6 & $<0.001$ \\
\hline East vs West & 0.007 & 0.033 & & NS & NS & \\
\hline
\end{tabular}

2) All CHD events (CHD death or definite or possible nonfatal MI). Logarithmic transformation of total triglycerides was performed because of skewed distribution in all statistical analyses including these variables.

\section{Results}

Table 1 reports the characteristics of the study populations according to sex. Subjects with NIDDM were older, more obese, and more often hypertensive than control subjects. With respect to smoking no difference between diabetic and non-diabetic subjects was observed. No East-West differences were found in age or smoking status. Plasma glucose was higher in diabetic men from East Finland than in West Finland. Both non-diabetic and diabetic women from East Finland were more obese than corresponding subjects from West Finland. Hypertension was more frequent in diabetic men and non-diabetic women from East Finland than in corresponding subjects from West Finland.

During the 7-year follow-up (mean follow-up was 7.2 years in men and women) 51 non-diabetic subjects ( 36 men, 15 women) and 163 diabetic patients (83 men, 80 women) from East Finland, and 30 nondiabetic subjects ( 21 men, 9 women) and 142 diabetic patients ( 88 men, 54 women) from West Finland died. Altogether 23 non-diabetic subjects ( 21 men, 2 women) and 120 diabetic patients $(62$ men, 58 women) from East Finland and 15 non-diabetic subjects (14 men, 1 woman) and 88 diabetic patients (55 men, 33 women) from West Finland died of cardiovascular causes (International Classification of Diseases 9, codes 390-458). CHD was the cause of death in 16 non-diabetic subjects ( 14 men, 2 women) and 92 diabetic subjects (53 men, 39 women) from East Finland, and 11 non-diabetic subjects (all men) and 66 diabetic subjects ( 44 men, 22 women) from West Finland. Thus, the percentage of subjects who died of CHD was $31 \%$ in non-diabetic and $56 \%$ in diabetic subjects from East Finland, and $37 \%$ in non-diabetic and $46 \%$ in diabetic subjects from West Finland of all subjects who died during the follow-up.

Table 2 reports age-adjusted rates of total mortality, cardiovascular mortality, and CHD mortality and morbidity in patients with NIDDM and non-diabetic subjects. Total and cardiovascular mortality rates were substantially higher in NIDDM patients than in non-diabetic subjects $(p<0.001)$. East-West difference in cardiovascular mortality was observed only in diabetic patients. In non-diabetic men, CHD mortality rates were 4.5 and $3.2 \%$ in East and West Finland, respectively. In both regions diabetic men had substantially higher rates of CHD mortality 


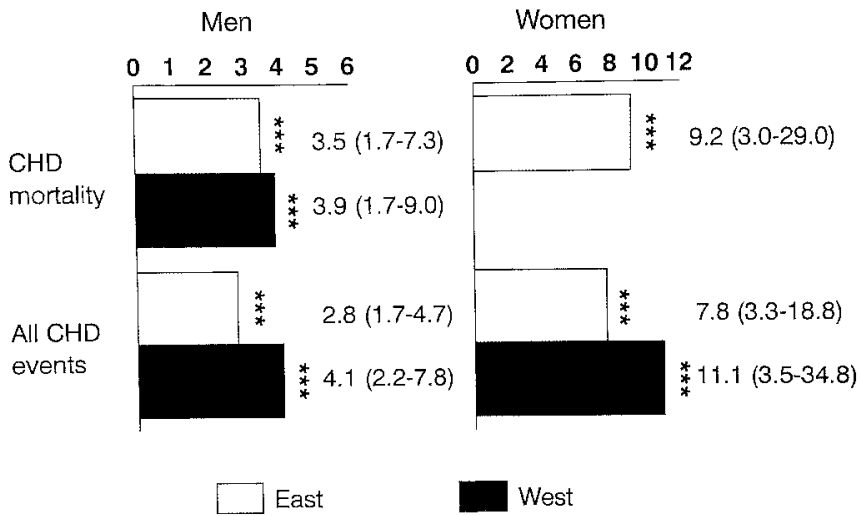

Fig. 1. Odds ratios (diabetic vs non-diabetic) and their $95 \%$ confidence intervals (in parentheses) for coronary heart disease (CHD) mortality and all CHD events (CHD mortality or non-fatal myocardial infarction) in East and West Finland by $\operatorname{sex}(* * * * 00.001)$

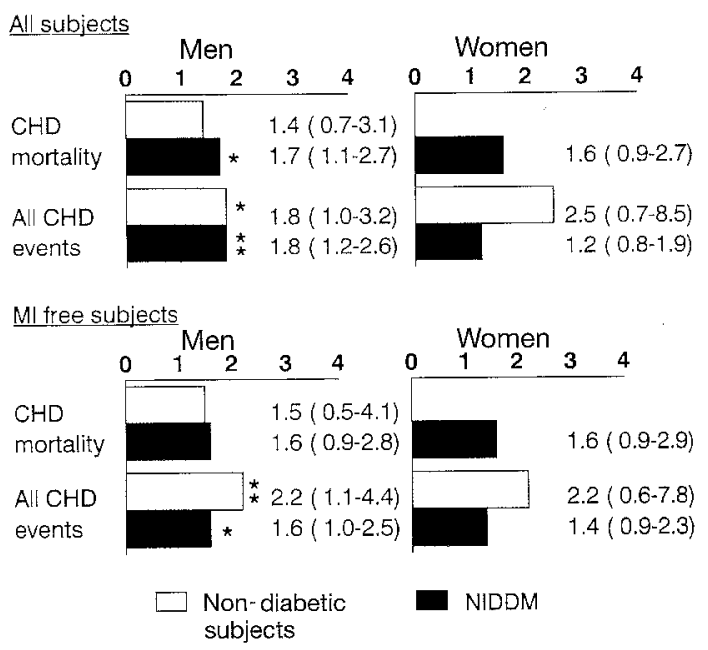

Fig. 2. Odds ratios (East vs West Finland) and their $95 \%$ confidence intervals (in parentheses) for coronary heart disease (CHD) mortality and all CHD events (CHD mortality or non-fatal myocardial infarction) for all subjects and for subjects free of myocardial infarction (MI) at baseline in non-diabetic and diabetic subjects by sex $\left({ }^{*} p<0.05,{ }^{*} p<0.01\right)$

$(p<0.001)$. In non-diabetic women, CHD mortality rates were much lower than those in men. NIDDM increased CHD mortality significantly in women $(p<0.001)$. Altogether $5.7 \%$ of non-diabetic men from West Finland and $10.0 \%$ of non-diabetic men from East Finland had either a MI or died of CHD during the follow-up. The corresponding percentages for non-diabetic women were 0.5 and $1.8 \%$, respectively. Diabetic men $(20.1 \%)$ and diabetic women $(11.5 \%)$ from West Finland and from East Finland ( 29.9 and $17.6 \%$, respectively) had significantly higher rates of CHD events than corresponding control subjects $(p<0.001)$. The results remained essentially similar although subjects with a history of MI before the baseline study were excluded. East-West difference was statistically significant only in men.
Figure 1 summarizes the odds ratios (diabetic vs non-diabetic) for CHD events by sex and area of residence. Diabetic men had 3-4 fold higher and diabetic women 8-11-fold higher risk for CHD than corresponding non-diabetic subjects $(p<0.001)$. Because none of the non-diabetic women from West Finland died from CHD during the follow-up odds ratios were not possible to calculate for this subgroup.

Figure 2 shows the odds ratios (East vs West) for CHD events in diabetic patients and non-diabetic control subjects by sex. Both non-diabetic and diabetic subjects had odds ratios of approximately 2 indicating a similar East-West difference in the CHD risk. In general, a regional difference was quite similar in men and women. The results remained similar when only MI-free subjects at baseline were included in statistical analyses.

\section{Discussion}

Our 7-year follow-up study based on data from two different non-diabetic and diabetic populations in Finland clearly demonstrated that the relative impact of NIDDM on the risk for CHD did not depend on the occurrence of CHD in non-diabetic populations of the same area. This indicates that diabetes per se and/ or factors associated with it promote atherothrombosis independently of cardiovascular risk factor levels or differences in the prevalence of CHD in non-diabetic population. Our study also confirms the results of several previous studies that NIDDM patients have a substantially greater risk of CHD events than non-diabetic subjects [18-24] and that the effect of NIDDM is particularly pronounced among women.

Although the occurrence of CHD and risk factors for CHD in NIDDM have been extensively studied, information is scarce on the effects of NIDDM on $\mathrm{CHD}$ risk in populations differing with respect of the occurrence of atherosclerotic complications. The International Atherosclerosis Project, an autopsy study carried out in 13 populations with varying occurrence of CHD showed that the variation in the extent of coronary atherosclerosis in diabetic patients between populations followed the same pattern as in non-diabetic subjects [25]. Thus, in populations with low occurrence of coronary atherosclerosis in nondiabetic subjects, the extent of coronary atherosclerosis in diabetic subjects also tended to be low, and vice versa. The drawback of this study was, however, that diabetic subjects were not classified into the two main types of the disease. Cross-sectional data from the WHO Multinational Study on Vascular Disease in Diabetics, based on 14 groups of diabetic subjects from different populations demonstrated a marked variation in the prevalence of CHD between diabetic populations [26]. The effect of NIDDM on the $\mathrm{CHD}$ risk was not possible to quantitate in that study 
because of the lack of non-diabetic control population and a reliable classification of patients into insulin-dependent and non-insulin-dependent diabetes. Therefore, the present report is the first prospective population study in which the CHD risk attributable to NIDDM can be reliably estimated in populations differing with respect to the extent of atherosclerotic vascular complications. Particular attention was paid to the selection of diabetic patients. Insulin-dependent diabetes was excluded on the basis of a history of ketoacidosis and glucagon-stimulated C-peptide measurements. Furthermore, both our NIDDM patients and non-diabetic control subjects were representative of the non-diabetic and diabetic population aged 45-64 years at baseline examination in both areas. Because odds ratios can give erroneous results if either diabetic or non-diabetic study populations are not true random samples it is important that the study populations are representative.

Our prospective study gave quite similar estimates for East-West difference with respect to CHD events in both baseline study (definite or possible $\mathrm{MI}$ in non-diabetic men: $1.3(0.8-2.3)$ and diabetic men: $2.0(1.3-3.0)$, definite or possible MI in non-diabetic women: $1.6(0.6-4.1)$ and diabetic women: $1.7(1.0$ 3.1) ) [9] and in this follow-up study (Fig. 2). However, East-West difference tended to be somewhat greater in non-diabetic subjects with respect to all CHD events than in NIDDM patients in the followup study (Fig. 2) which may indicate that the relative impact of NIDDM with respect to the risk of CHD decreases with the duration of the disease.

In our study risk factor levels were significantly more adverse in East Finland than in West Finland (Table 1) in both non-diabetic and diabetic populations. The only East-West difference in NIDDM patients which was not observed in the non-diabetic population was in total triglyceride level in women. Furthermore, East-West differences in other cardiovascular risk factor levels seemed to be proportionally more adverse among diabetic patients than in nondiabetic control subjects. However, the relative risk for CHD in NIDDM patients tended to be somewhat lower in the high CHD area. Our results are in agreement with the findings reported by Stamler et al. [24] from the Multiple Risk Factor Intervention Trial. Among 5163 men who reported taking medication for diabetes, the risk for CHD mortality was relatively lower compared to that of 342,815 non-diabetic men with high risk factors levels than with low risk factor levels during 12-year follow-up. However, absolute risk of CHD increased more steeply among diabetic men than among non-diabetic men. The low number of CHD deaths among non-diabetic subjects (particularly women) prevented us from carrying out similar statistical analyses of our data as well as analyses on risk factors for CHD which might explain the East-West difference.
The main result of our study is that the impact of NIDDM on the incidence of CHD is so strong that it becomes manifest with a similar force in populations with high (East Finland) and relatively low (West Finland) risk for atherothrombosis; this provided important information with respect to the aetiology of CHD in NIDDM. These results imply that factors related to NIDDM, independently of conventional cardiovascular risk factors and the occurrence of atherosclerotic complications in the background population, must play a major role in the pathogenesis of macrovascular disease in NIDDM. These factors include at least hyperinsulinaemia, hyperglycaemia, enhanced thrombus formation, modification of lipids and lipoproteins and lipoprotein composition [27]. Indeed, our recent data from another epidemiological study provides evidence that glycated haemoglobin $A_{1 c}$ and glucose levels are strong risk factors for CHD in elderly subjects with NIDDM [28]. Therefore, factors associated with NIDDM itself should be given more attention in future studies aiming to investigate the determinants of CHD in this type of diabetes.

Acknowledgements. This study was supported by a grant from the Academy of Finland.

\section{References}

1. Pyörälä K, Laakso M, Uusitupa M (1987) Diabetes and atherosclerosis: an epidemiological view. Diabetes Metab Rev 3: 463-524

2. Keys A (1970) Coronary heart disease in seven countries. Circulation 41/42 [Suppl I]: 1-211

3. Pyörälä K (1987) Interpopulation correlations between serum cholesterol level and the occurrence of coronary heart disease. Eur Heart J 8 [Suppl E]: 23-30

4. Pyörälä K, Epstein FH, Kornitzer M (1985) Changing trends in coronary heart disease mortality: possible explanations. Cardiology 72: 5-10 [Editorial]

5. Salomaa V, Arstila M, Kaarsalo E et al. (1992) Trends in the incidence of and mortality from coronary heart disease in Finland from 1983 to 1988. Am J Epidemiol 136: 13031315

6. Pyörälä K, Salonen JT, Valkonen T (1985) Trends in coronary heart disease mortality and morbidity and related factors in Finland. Cardiology 72: 35-51

7. Vartiainen E, Korhonen HJ, Pietinen P et al. (1991) Fifteen-year trends in coronary risk factors in Finland, with special reference to North Karelia. Int J Epidemiol 20: 651-662

8. De la Chapelle A (1993) Disease gene mapping in isolated human populations: the example of Finland. J Med Genet 30: $857-865$

9. Laakso M, Rönnemaa T, Pyörälä K, Kallio V, Puukka P, Penttilä I (1988) Atherosclerotic vascular disease and its risk factors in non-insulin-dependent diabetic and nondiabetic subjects in Finland. Diabetes Care 11: 449-463

10. Madsbad S, Alberti KGMM, Binder C, Burrin JM, Faber OK, Krarup T, Regeus L (1979) Role of residual insulin secretion in protecting against ketoacidosis in insulin-dependent diabetics. BMJ 2: 1257-1259 
11. World Health Organization (1985) Diabetes mellitus: report of a WHO study group. Geneva, World Health Org Tech Rep Ser, no727

12. World Health Organization (1983) Proposal for the multinational monitoring of trends and determinants in cardiovascular disease and protocol (MONICA Project). Geneva, World Health Organization (WHO/MNC/82.1 Rev. 1)

13. Faber OK, Binder C (1977) C-peptide response to glucagon: a test for the recidual beta-cell function in diabetes mellitus. Diabetes 26: 605-610

14. Kostner GM (1976) Enzymatic determination of cholesterol in high density lipoprotein fractions prepared by polyanion precipitation. Clin Chem 22: 695-698

15. Armitage P (1971) Statistical methods in medical research. Blackwell Oxford, UK

16. Searle SR (1971) Linear models. Wiley, New York

17. Gart J (1962) On the combination of relative risks. Biometrics 18: 601-610

18. Panzram G (1987) Mortality and survival in type 2 (non-insulin-dependent) diabetes mellitus. Diabetologia 30: 123131

19. Barrett-Connor E, Wingard DL (1983) Sex differential in ischemic heart disease mortality in diabetics: a prospective population based study. Am J Epidemiol 118: 489-496

20. Butler WJ, Ostrander LD, Jr, Carman WJ, Lamphiear DE (1985) Mortality from coronary heart disease in Tecumseh study: long term effect of diabetes mellitus, glucose tolerance and other risk factors. Am J Epidemiol 121: 541-547

21. Kannel WB, McGee DL (1979) Diabetes and cardiovascular risk factors in the Framingham Study. Circulation 59: 8-13
22. Fuller JH, Shipley MJ, Rose J, Jarrett RJ, Keen H (1983) Mortality from coronary heart disease and stroke in relation to degree of hyperglycemia: the Whitehall study. BMJ 27: $867-870$

23. Morrish NJ, Stevens LK, Head J, Fuller JH, Jarrett RJ, Keen $H$ (1990) A prospective study of mortality among middle-aged diabetic patients (the London cohort of the WHO Multinational Study of Vascular Disease in Diabetes). II: associated risk factors. Diabetologia 33: 543-548

24. Stamler J, Vaccaro O, Neaton JD, Wentworth D, for the Multiple Risk Factor Intervention Trial Diabetes, other risk factors, and 12-yr cardiovascular mortality for men screened in the Multiple Risk Factor Intervention Trial. Diabetes Care 16: 434-444

25. Robertson WB, Strong JP (1968) Atherosclerosis in persons with hypertension and diabetes mellitus. Lab Invest 18: 538-551

26. Diabetes Drafting Group (1985) Prevalence of small vessel and large vessel disease patients from 14 centres: the World Health Organization multinational study of vascular disease in diabetics. Diabetologia 28: 615-640

27. Bierman EL (1992) Atherogenesis in diabetes. Arterioscl Thromb 12: 647-656

28. Kuusisto J, Mykkänen L, Pyörälä K, Laakso M (1994) Noninsulin-dependent diabetes and its metabolic control predict coronary heart disease in elderly subjects. Diabetes 43: $960-967$ 